\title{
Paraaortic Region
}

National Cancer Institute

\section{Source}

National Cancer Institute. Paraaortic Region. NCI Thesaurus. Code C25316.

Regions near to the aorta. 